\title{
Fat and Fiber: How the Controlled Attenuation Parameter Complements Noninvasive Assessment of Liver Fibrosis
}

\author{
Grace Lai-Hung Wong • Vincent Wai-Sun Wong
}

Received: 2 November 2014/Accepted: 5 November 2014/Published online: 16 November 2014

(C) Springer Science+Business Media New York 2014

Nonalcoholic fatty liver disease (NAFLD) is an evergrowing problem in the developed and developing worlds, affecting 15-40\% of the population [1]. Once regarded as a benign condition, NAFLD is the leading cause of cryptogenic cirrhosis and cryptogenic hepatocellular carcinoma (HCC) [2], and the third most frequent indication for liver transplantation [3].

A key challenge faced by clinicians who manage NAFLD is to establish the diagnosis and to differentiate simple steatosis from advanced disease [4]. Furthermore, steatosis often coexists in other chronic liver diseases such as chronic hepatitis C [5]. Trans-abdominal ultrasonography (USG) is the commonest imaging tool used in the diagnosis of hepatic steatosis, but only with confidence when the amount of fatty infiltration exceeds $33 \%$ [6]. Although proton magnetic resonance spectroscopy $\left({ }^{1} \mathrm{H}-\mathrm{MRS}\right)$ is an accurate and reproducible noninvasive means of quantifying hepatic fat, its availability is limited in many areas [1].

Transient elastography (Fibroscan, Echosens, Paris, France), originally developed for the diagnosis of liver fibrosis and cirrhosis, has been validated in patients with NAFLD [7]. The fundamental technology employed by Fibroscan involves a plastic shear wave that propagates

G. L.-H. Wong $(\bowtie) \cdot$ V. W.-S. Wong

Institute of Digestive Disease, The Chinese University of Hong

Kong, Shatin, Hong Kong SAR, China

e-mail: wonglaihung@cuhk.edu.hk

G. L.-H. Wong · V. W.-S. Wong

Department of Medicine and Therapeutics, The Chinese

University of Hong Kong, Shatin, Hong Kong SAR, China

G. L.-H. Wong · V. W.-S. Wong

State Key Laboratory of Digestive Disease, The Chinese

University of Hong Kong, Shatin, Hong Kong SAR, China through liver tissue combined with a pulse-echo ultrasound used to follow and measure the velocity of the propagation of the shear wave, which is directly related to tissue stiffness and hence severity of liver fibrosis [8]. Its latest iteration includes the measurement of the controlled attenuation parameter (CAP), a measurement of the attenuation of the forward and return ultrasound signal at 3.5 $\mathrm{MHz}$ using signals acquired by the regular $\mathrm{M}$ probe of Fibroscan. At a given frequency, the ultrasound-attenuation coefficient can be expressed in $\mathrm{dB} / \mathrm{m}$. Since the attenuation of ultrasound signals is greater in fat than in water, CAP may be used to estimate the degree of hepatic steatosis [9]. The diagnostic attractiveness of CAP is that it may be measured concurrently with liver stiffness using the same instrument enabling the simultaneous assessment of hepatic steatosis and fibrosis [8].

Data concerning the accuracy of CAP to diagnosis different degrees of hepatic steatosis are evolving. CAP efficiently detected low-grade steatosis ( $>10 \%)$, with a sensitivity of $91 \%$ and specificity of $81 \%$ at a cutoff value of $238 \mathrm{~dB} / \mathrm{m}$ in a retrospective cohort of patients of mixed etiology chronic liver diseases [9]. The accuracy of CAP was confirmed in two prospective studies of mixed etiology liver disease $[10,11]$, and in single etiology populations, including chronic hepatitis $\mathrm{B}(\mathrm{CHB})$, chronic hepatitis $\mathrm{C}$, NAFLD, and alcoholic liver disease [12]. The suggested diagnostic performance and cutoff values for different degrees of steatosis are summarized in Table 1. Nonetheless, data concerning its performance in healthy subjects and patients with $\mathrm{CHB}$ were either lacking or insufficient.

In this issue of the Digestive Diseases and Sciences, two groups of investigators provide important data to illustrate the accuracy of CAP in these two settings. Chon et al. [16] report CAP data from 264 healthy subjects, either as potential liver donors or in subjects undergoing routine 


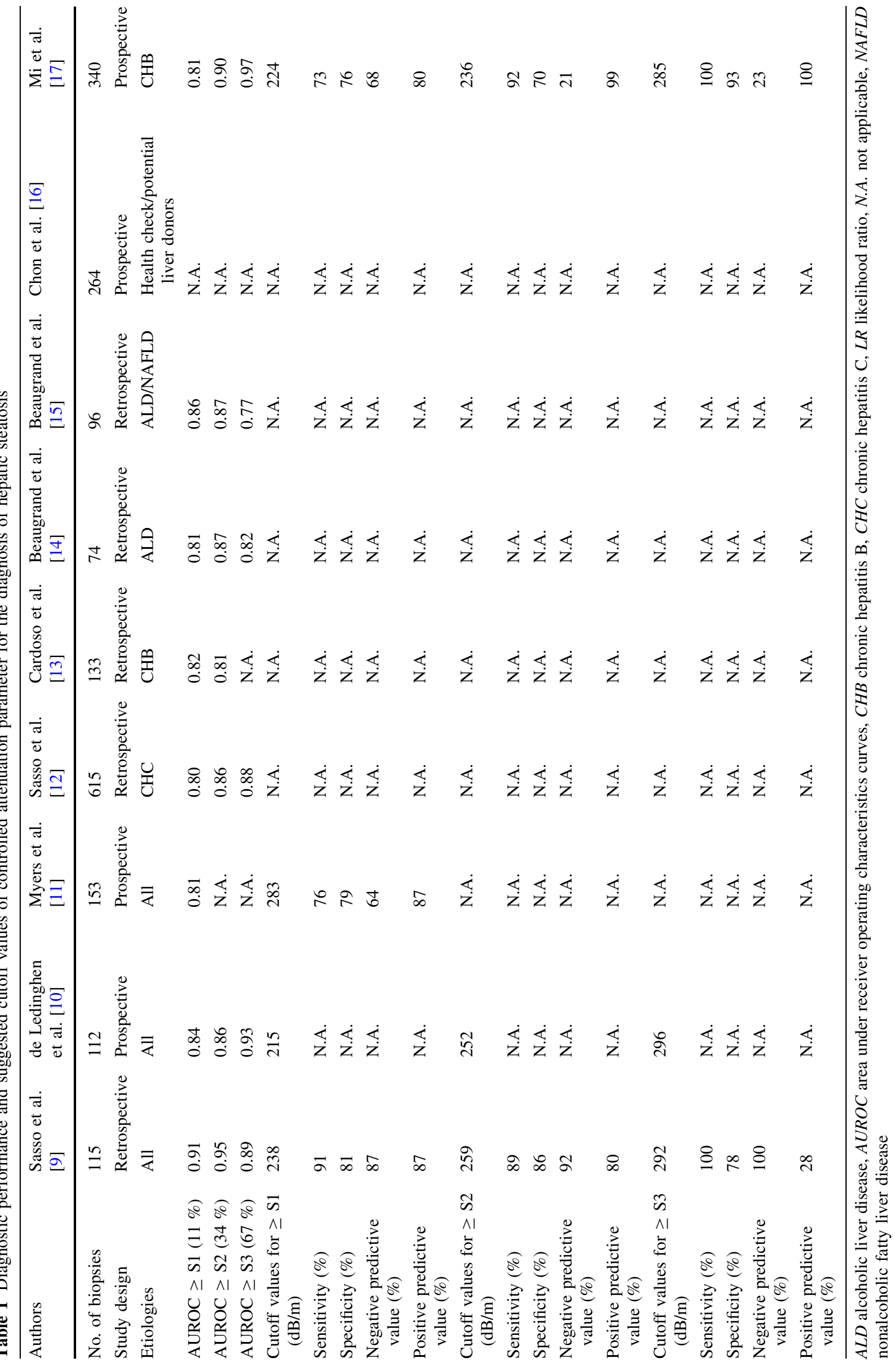


health assessment. They defined the range of normal CAP values (5th-95th percentiles) as $156.0-287.8 \mathrm{~dB} / \mathrm{m}$. Interestingly, the mean CAP value was slightly yet significantly higher in the healthy group than in the potential liver donor group $(227.5 \pm 42.0$ vs. $218.2 \pm 28.3 \mathrm{~dB} / \mathrm{m}, P=0.040)$. Higher body mass index (BMI) and serum triglyceride concentrations were associated with higher CAP values of healthy subjects, implying that there were some NAFLD subjects in the routine assessment group, in which 9.0 and $4.3 \%$ of subjects had metabolic syndrome and diabetes mellitus, respectively. Hence, the stated upper limit of normal (ULN) for CAP values, $287.8 \mathrm{~dB} / \mathrm{m}$, is likely too high. Instead, $266.3 \mathrm{~dB} / \mathrm{m}$, the ULN for CAP in potential liver donors, was probably a more reasonable value. According to previous publications, the range for lowgrade steatosis ( $>10 \%$ fatty infiltration) is $215-283 \mathrm{~dB} / \mathrm{m}$ [8].

In the same issue, $\mathrm{Mi}$ et al. report their validation study in $340 \mathrm{CHB}$ patients who underwent liver biopsy [17], by far one of the largest CAP studies in this population. This important study provides not only data on the accuracy of CAP but also information on potential confounders. This study unequivocally reassures us that necroinflammation, as represented by serum alanine aminotransferase (ALT) and the Knodell histological activity index, does not influence CAP. Instead, only BMI and steatosis grade correlated positively with CAP. Thus, while a high ALT level independently increases liver stiffness and can contribute toward a false diagnosis of cirrhosis [18], CAP does not suffer from the same confounding issues.

As pointed out by the authors, higher CAP cutoff values in subjects with higher BMI were also reported in several other studies [10], perhaps reflecting excess attenuation due to fat overlying the liver. To overcome this confounder, the skin-liver capsule distance (SCD) was applied to multivariate analysis; $\mathrm{SCD}>25 \mathrm{~mm}$ consistently increased CAP $\sim 60 \mathrm{~dB} / \mathrm{m}$ regardless of the grade of hepatic steatosis (unpublished data from the same group). Therefore, a SCDbased algorithm, similar to the ALT-based algorithm for LSM derived from CHB patients [19], may be considered for the interpretation of CAP in obese subjects.

The clinical significance of diagnosing fatty liver in CHB patients is somewhat obscure. Although $\mathrm{CHB}$ is associated with a lower prevalence of fatty liver [20], the metabolic syndrome increases the risk of cirrhosis and fibrosis progression in CHB patients [21, 22]. It would thus be interesting to study if CAP is prognostically important in addition to liver stiffness and other traditional risk factors of hepatocellular carcinoma and cirrhotic complications [23].

To sum up, these two important studies established the diagnostic accuracy of CAP for hepatic steatosis in different settings. Since CAP and liver stiffness can be measured simultaneously, transient elastography be used in multiple clinical settings, providing comprehensive yet convenient noninvasive assessment of the liver of considerable value in the selection of candidates for treatment.

Conflict of interest Grace Wong has served as an advisory committee member for Otsuka and Gilead. She has also served as a speaker for Abbvie, Bristol-Myers Squibb, Echosens, Furui, Gilead, Janssen, and Otsuka. Vincent Wong has served as an advisory committee member for Abbvie, Roche, Novartis, Gilead, and Otsuka. He has also served as a speaker for Abbvie, Bristol-Myers Squibb, Roche, Novartis, Abbott Diagnostics, and Echosens.

\section{References}

1. Wong VW, Chu WC, Wong GL, et al. Prevalence of non-alcoholic fatty liver disease and advanced fibrosis in Hong Kong Chinese: a population study using proton-magnetic resonance spectroscopy and transient elastography. Gut. 2012;61:409-415.

2. Wong VW, Wong GL, Choi PC, et al. Disease progression of non-alcoholic fatty liver disease: a prospective study with paired liver biopsies at 3 years. Gut. 2010;59:969-974.

3. Charlton MR, Burns JM, Pedersen RA, et al. Frequency and outcomes of liver transplantation for nonalcoholic steatohepatitis in the United States. Gastroenterology. 2011;141:1249-1253.

4. Chan HL, de Silva HJ, Leung NW, et al. How should we manage patients with non-alcoholic fatty liver disease in 2007? J Gastroenterol Hepatol. 2007;22:801-808.

5. Clouston AD, Jonsson JR, Powell EE. Steatosis as a cofactor in other liver diseases: hepatitis $\mathrm{C}$ virus, alcohol, hemochromatosis, and others. Clin Liver Dis. 2007;11:173-189, x.

6. Saadeh S, Younossi ZM, Remer EM, et al. The utility of radiological imaging in nonalcoholic fatty liver disease. Gastroenterology. 2002;123:745-750.

7. Wong VW, Vergniol J, Wong GL, et al. Diagnosis of fibrosis and cirrhosis using liver stiffness measurement in nonalcoholic fatty liver disease. Hepatology. 2010;51:454-462.

8. Wong GL. Transient elastography: kill two birds with one stone? World J Hepatol. 2013;5:264-274.

9. Sasso M, Beaugrand M, de Ledinghen V, et al. Controlled attenuation parameter (CAP): a novel VCTE guided ultrasonic attenuation measurement for the evaluation of hepatic steatosis: preliminary study and validation in a cohort of patients with chronic liver disease from various causes. Ultrasound Med Biol. 2010;36:1825-1835.

10. de Ledinghen V, Vergniol J, Foucher J, et al. Non-invasive diagnosis of liver steatosis using controlled attenuation parameter (CAP) and transient elastography. Liver Int. 2012;32:911-918.

11. Myers RP, Pollett A, Kirsch R, et al. Controlled Attenuation Parameter (CAP): a noninvasive method for the detection of hepatic steatosis based on transient elastography. Liver Int. 2012;32:902-910.

12. Sasso M, Tengher-Barna I, Ziol M, et al. Novel controlled attenuation parameter for noninvasive assessment of steatosis using Fibroscan $((\mathrm{R}))$ : validation in chronic hepatitis C. J Viral Hepat. 2012;19:244-253.

13. Cardoso AC, Sasso M, Miette V, et al. Controlled attenuation parameter: a novel FibroScan-based tool to detect and quantify steatosis in chronic hepatitis B. http://onlinelibrary.wiley.com. easyaccess1.lib.cuhk.edu.hk/doi/10.1002/hep.23974/pdf.

14. Beaugrand M, de Ledinghen V, Douvin C, et al. Validation of controlled attenuation parameter (CAP) as a non-invasive marker 
of steatosis in 228 patients with chronic liver disease from various causes. J Hepatol. 2010;52:S35-S36.

15. Beaugrand M, Ziol M, de Ledinghen V, et al. Controlled attenuation parameter: A novel FibroScan ${ }^{\circledR}$-based tool to detect and quantify steatosis. Preliminary study in patient with alcoholoc and non alcoholic fatty liver disease. J Hepatol. 2010;52:S158S159.

16. Chon YE, Jung KS, Kim KJ, et al. Normal controlled attenuation parameter values: a prospective study of healthy subjects undergoing health checkups and liver donors in Korea. Dig Dis Sci. (Epub ahead of print). doi:10.1007/s10620-014-3293-1.

17. Mi YQ, Shi QY, Zu L, et al. Controlled attenuation parameter for noninvasive assessment of hepatic steatosis using Fibroscan $\left({ }^{\circledR}\right)$ : validation in chronic hepatitis B. Dig Dis Sci. (Epub ahead of print). doi:10.1007/s10620-014-3341-x.

18. Wong GL, Wong VW, Choi PC, et al. Increased liver stiffness measurement by transient elastography in severe acute exacerbation of chronic hepatitis B. J Gastroenterol Hepatol. 2009;24: 1002-1007.
19. Chan HL, Wong GL, Choi PC, et al. Alanine aminotransferasebased algorithms of liver stiffness measurement by transient elastography (Fibroscan) for liver fibrosis in chronic hepatitis B. $J$ Viral Hepat. 2009;16:36-44.

20. Wong VW, Wong GL, Chu WC, et al. Hepatitis B virus infection and fatty liver in the general population. J Hepatol. 2012;56: 533-540.

21. Wong GL, Chan HL, Yu Z, et al. Coincidental metabolic syndrome increases the risk of liver fibrosis progression in patients with chronic hepatitis B-a prospective cohort study with paired transient elastography examinations. Aliment Pharmacol Ther. 2014;39:883-893.

22. Wong GL, Wong VW, Choi PC, et al. Metabolic syndrome increases the risk of liver cirrhosis in chronic hepatitis B. Gut. 2009;58:111-117.

23. Wong GL, Chan HL, Wong CK, et al. Liver stiffness-based optimization of hepatocellular carcinoma risk score in patients with chronic hepatitis B. J Hepatol. 2014;60:339-345. 